\title{
NONLINEAR STABILITY OF TWO-LAYER FLOWS *
}

\author{
PAUL MILEWSKI ${ }^{\dagger}$, ESTEBAN TABAK ${ }^{\ddagger}$, CRISTINA TURNER §, RUBEN ROSALES `, \\ AND FERNANDO MENZAQUE \|
}

\begin{abstract}
We study the dynamics of two-layer, stratified shallow water flows. This is a model in which two scenarios for eventual mixing of stratified flows (shear-instability and internal breaking waves) are, in principle, possible. We find that unforced flows cannot reach the threshold of shearinstability, at least without breaking first. This is a fully nonlinear stability result for a model of stratified, sheared flow. Mathematically, for 2X2 autonomous systems of mixed type, a criterium is found deciding whether the elliptic domain is reachable-smoothly-from hyperbolic initial conditions. If the characteristic fields depend smoothly on the system's Riemann invariants, then the elliptic domain is unattainable. Otherwise, there are hyperbolic initial conditions that will lead to incursions into the elliptic domain, and the development of the associated instability.
\end{abstract}

Key words. shear instability, systems of mixed type, two-layer flows, shallow water.

AMS subject classification: 35M10, 76E20, 76B55.

\section{Introduction}

We study the dynamics of two-layer, shallow water flows. This dynamics in principle includes two closely related physical scenarios for mixing: internal breaking waves and shear instability. Even though the rolls arising from shear instability are visually reminiscent of breaking waves, the two phenomena are clearly distinct. Mathematically, breaking waves arise from nonlinear wave modulation in hyperbolic systems, while instability can be associated with the equations governing the dynamics turning elliptic $[1,2]$. Physically, wave propagation is primarily a conservative process that concentrates energy at the location of breaking, whereas shear instability is a process that creates vortical structures by withdrawing energy from the mean flow. Thus, for mathematical models of stratified fluids allowing in principle both breaking waves and excursions into elliptic domains, we may ask the following natural question: with smooth initial data in the stable (hyperbolic) regime, are the two scenarios attainable? Or does wave breaking always precede and possibly preclude shear instability? In mathematical terms, we are asking under which conditions can systems of mixed type, given data in the hyperbolic domain, actually reach their elliptic domain.

In order to make progress on this question, we concentrate here on what is possibly its simplest setting: the one-dimensional evolution of long waves in two-layer flows of slightly different density, constrained by two horizontal rigid lids [3]. This problem leads to an autonomous system of two equations of mixed type. In this context, we find a precise nonlinear stability criterion, and confirm it with numerical experiments. We find that unforced two-layer flows remain hyperbolic, at least as long as the flow remains smooth. Hence, in our model, breaking waves always precede, and maybe altogether eliminate, shear instability. Beyond fluid dynamics, for general $2 \times 2$ autonomous systems of mixed type, the elliptic domain is unreachable if its

\footnotetext{
*Received: May 14, 2004; accepted (in revised version): August 7, 2004. Communicated by John Hunter.

${ }^{\dagger}$ Deptartment of Mathematics, University of Wisconsin, Madison.

$\ddagger$ Courant Institute, New York University.

$\S$ Famaf, Universidad Nacional de Cordoba.

IDeptartment of Mathematics, Massachusetts Institute of Technology.

" Famaf, Universidad Nacional de Cordoba.
} 
characteristic speeds are smooth functions of the Riemann invariants.

Hence, at least for shallow water two-layer flows, shear-instability can only arise from sources external to the flow, through external forces or boundary conditions. Then the only mechanism by which the system's internal dynamics may lead to mixing is through breaking waves. This contrasts sharply with the way mixing is typically conceptualized and modeled in present-day general circulation models [4]. There mixing results solely from shear-instability, parameterized by the Richardson number $R i$, which measures the relative strengths of the stratification, stabilizing the flow, to the shear, which renders it unstable $[5,6]$.

\section{Two layer hydraulics}

Consider two layers of incompressible fluid between two horizontal rigid lids (see figure 2). We shall denote the lower layer 1 and the upper layer 2, with respective (constant) densities $\rho_{1}$ and $\rho_{2}$, mean horizontal velocities $u_{1}$ and $u_{2}$, and thicknesses $h_{1}$ and $h_{2}$, with $h_{1}+h_{2}=H$, where $H$ is the distance between the two rigid boundaries. The pressure at the top rigid lid will be denoted $P$. The unknowns are therefore $h_{1}$, $h_{2}, P, u_{1}$ and $u_{2}$, all functions of $(x, t)$. We shall consider the usual shallow water limit where the pressure is hydrostatic and the flow is primarily in $x$.

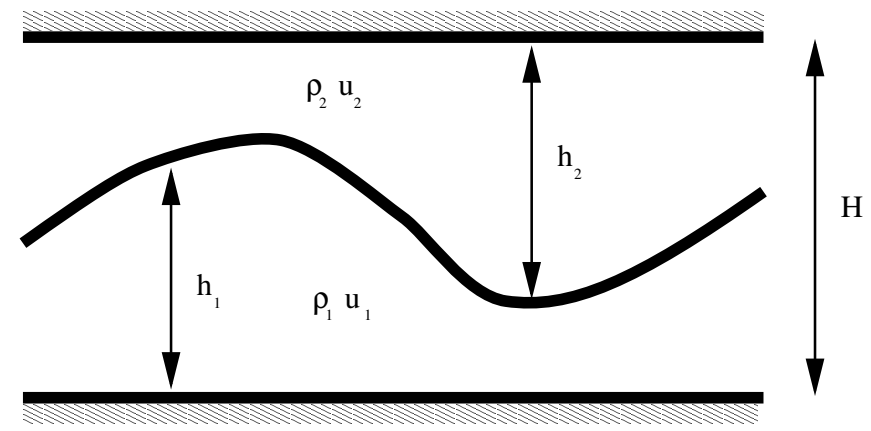

FIG. 2.1. Two-layered fluid model

The corresponding equations describing the motion are:

$$
\begin{aligned}
& \left(h_{2}\right)_{t}+\left(h_{2} u_{2}\right)_{x}=0 \\
& \left(h_{1}\right)_{t}+\left(h_{1} u_{1}\right)_{x}=0
\end{aligned}
$$

corresponding to conservation of mass and volume for the two layers, and

$$
\begin{array}{r}
\left(h_{2} u_{2}\right)_{t}+\left(h_{2} u_{2}^{2}+\frac{P h_{2}}{\rho_{2}}+g \frac{h_{2}^{2}}{2}\right)_{x}=-\frac{1}{\rho_{2}}\left(P+g \rho_{2} h_{2}\right) h_{1 x} \\
\left(h_{1} u_{1}\right)_{t}+\left(h_{1} u_{1}^{2}+\frac{\left(P+g \rho_{2} h_{2}\right) h_{1}}{\rho_{1}}+g \frac{{h_{1}}^{2}}{2}\right)_{x}=\frac{1}{\rho_{1}}\left(P+g \rho_{2} h_{2}\right) h_{1 x},
\end{array}
$$

for conservation of momentum.

Adding (2.1) and (2.2) yields the constraint

$$
\left(h_{1} u_{1}+h_{2} u_{2}\right)=Q(t)
$$


Adding (2.3) and (2.4), on the other hand, yields an equation relating $P$ and $Q^{\prime}$ :

$$
P_{x}+\left(\frac{h_{1}}{\rho_{1}}+\frac{h_{2}}{\rho_{2}}\right)^{-1}\left[\left(h_{1} u_{1}^{2}+h_{2} u_{2}^{2}\right)_{x}+g^{\prime} h_{1} h_{1 x}+Q^{\prime}(t)\right]=0,
$$

where $g^{\prime}$ is the reduced gravity

$$
g^{\prime}=g \frac{\rho_{1}-\rho_{2}}{\rho_{1}} .
$$

The method for solving $(2.5,2.6)$ for $P$ and $Q$ depends on the boundary conditions. If there is a boundary such that there is no flux at a given point (such as a fixed sidewall), then $Q(t)=0$, and $P(x, t)$ follows simply from integrating (2.6) (with an arbitrary constant of integration with no physical relevance, since only $P_{x}$ appears in the equations). If the boundary conditions are periodic, on the other hand, then integrating (2.6) over the full domain yields $Q^{\prime}(t)$ :

$$
Q^{\prime}(t)=-\frac{\int_{0}^{L}\left(\frac{h_{1}}{\rho_{1}}+\frac{h_{2}}{\rho_{2}}\right)^{-1}\left[\left(h_{1} u_{1}^{2}+h_{2} u_{2}^{2}\right)_{x}+g^{\prime} h_{1} h_{1 x}\right] d x}{\int_{0}^{L}\left(\frac{h_{1}}{\rho_{1}}+\frac{h_{2}}{\rho_{2}}\right)^{-1} d x},
$$

and then $P$ follows again from integrating (2.6).

In either case, this allows us to think of the system $(2.1,2.2,2.3,2.4)$ as describing the evolution of two dynamical variables, say $u_{1}$ and $h_{1}$, together with two quantities, $P(x, t)$ and $Q(t)$, which follow from global constraints. For example, one can use equations (2.2) and (2.4) to evolve $u=u_{1}$ and $h=h_{1}$, with $h_{2}=H-h_{1}, u_{2}$ given by (2.5), $P$ by (2.6), and $Q$ updated in the periodic case using (2.8), or set to zero in the bounded case.

We can rewrite (2.2) and (2.4) in the form

$$
\begin{array}{r}
h_{t}+u h_{x}+h u_{x}=0 \\
u_{t}+u u_{x}+\frac{P_{x}}{\rho_{1}}+g^{\prime} h_{x}=0 .
\end{array}
$$

In order to obtain a simpler model, we shall invoke the Boussinesq assumption, whereby density variations are small, and become negligible in the inertial terms. This allows us to take $Q(t)=0$ even in the periodic case, since $(2.8)$ yields $Q^{\prime}(t)=0$ $\left(h_{1} / \rho_{1}+h_{2} / \rho_{2}\right.$ is replaced by $H / \rho_{1}$, and the rest is the integral of derivatives of periodic functions over a period.)

Then, with $Q=0, P_{x}$ from (2.6) is given by

$$
\frac{P_{x}}{\rho_{1}}=-\left[\frac{h}{H-h} u^{2}+g^{\prime} \frac{h^{2}}{2 H}\right]_{x},
$$

and the system $(2.9,2.10)$ becomes

$$
\begin{aligned}
h_{t}+u h_{x}+h u_{x} & =0 \\
u_{t}+\frac{H-3 h}{H-h} u u_{x}+\left(g^{\prime} \frac{H-h}{H}-\frac{H}{(H-h)^{2}} u^{2}\right) h_{x} & =0 .
\end{aligned}
$$


We shall nondimensionalize the system $(2.12,2.13)$, by making the replacement

$$
\begin{aligned}
h & \rightarrow H h \\
u & \rightarrow \sqrt{g^{\prime} H} u \\
x & \rightarrow H x \\
t & \rightarrow \sqrt{\frac{H}{g}} t,
\end{aligned}
$$

so that it becomes

$$
\begin{aligned}
h_{t}+u h_{x}+h u_{x} & =0 \\
u_{t}+\frac{1-3 h}{1-h} u u_{x}+\left((1-h)-\frac{1}{(1-h)^{2}} u^{2}\right) h_{x} & =0 .
\end{aligned}
$$

These equations, describing the flow of two fluid layers of slightly different density between two horizontal rigid boundaries, are those we shall study in the remaining sections.

\section{Hyperbolic and elliptic domains: instabilities and mixing}

The eigenvalues of the system $(2.18,2.19)$ are given by

$$
\lambda=\frac{1-2 h}{1-h} u \pm \sqrt{h \frac{(1-h)^{2}-u^{2}}{1-h}},
$$

so the system is of mixed type: hyperbolic when

$$
\frac{(1-h)^{2}}{u^{2}}>1
$$

and elliptic otherwise. We define the quantity

$$
R i=\frac{(1-h)^{2}}{u^{2}},
$$

the Richardson number for two-layer flows. In dimensional variables, this takes the form

$$
R i=\frac{g^{\prime}(H-h)^{2}}{H u^{2}}
$$

and the condition for hyperbolicity of the system $(2.12,2.13)$ is still that $R i>1$.

This Richardson number has a simple interpretation in terms of the ratio of the kinetic energy available for mixing the two layers, to the potential energy barrier given by the stratification [7]. To see this, consider a complete mixing scenario, in which we start with two distinct layers, and end up with a single, homogeneous one with density $\rho^{*}$ and velocity $u^{*}=0$ (see 3 ).

Conservation of mass yields

$$
\rho^{*}=\frac{\rho_{1} h+\rho_{2}(H-h)}{H} .
$$

The gain in the potential energy

$$
P E=\int_{0}^{H} g \rho z d z
$$




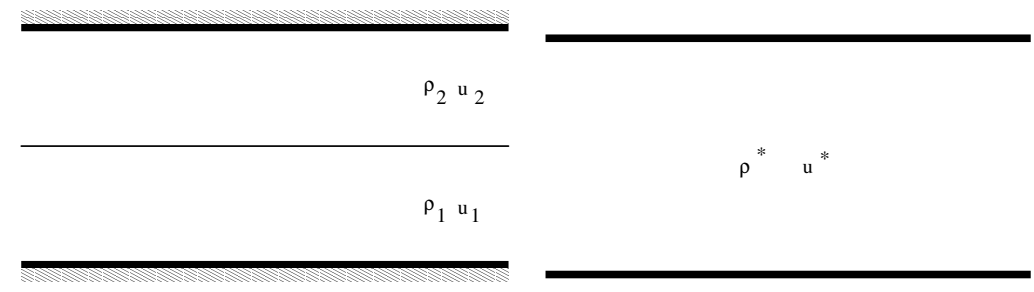

FIG. 3.1. The two layers and the fully mixed fluid

is given by

$$
P E_{+}-P E_{-}=\frac{1}{2} g^{\prime} \rho_{1} h(H-h),
$$

where the subindexes + and - refer to the states after and before the mixing process. The loss in the kinetic energy

$$
K E=\frac{1}{2} \int_{0}^{H} \rho_{1} u^{2} d z
$$

on the other hand, is given by

$$
K E_{-}-K E_{+}=\frac{1}{2} \rho_{1} \frac{H h}{H-h} u^{2} .
$$

Hence the quotient between the potential energy gain and the kinetic energy loss is given precisely by the Richardson number (3.3). Hence the elliptic regime $R i<1$ corresponds to a state in which the flow is sufficiently energetic that it could, for miscible fluids, mix completely the two layers.

This is intuitively appealing since in many applications in physics, when an initial value problem becomes elliptic, this is interpreted as an indication of an instability. Here, we could imagine a mixing process induced by Rayleigh-Taylor instabilities if the flow is sufficiently sheared. However, for the system $(2.18,2.19)$, we shall see that, for smooth solutions, if the flow is initially everywhere hyperbolic, it will so indefinitely. In figures 3.2, 3.3 we show numerical calculations of solutions before breaking; they never cross the sonic boundary $R i=1$.

It is worth noting that the configuration of figure 2, taken as initial data not for two-layer flows, but for the full 2D Euler equations, is unstable to shear for all values of $R i$, not just for $R i<1$ as in the two-layer model [1]. To interpret the results of the two-layer model for $R i>1$, one considers that, even though the real fluid system is unstable, the instability will only mix a thin layer between the two fluids and stabilize the system, leaving long-wave propagation and breaking as the dominant components of the system's dynamics. It is only when $R i<1$ that the instability has sufficient energy to fully mix the two layers into one, replacing wave propagation and breaking in their central dynamical role.

Clearly, if one is in the unstable regime, the shallow water approximations in equations $(2.18,2.19)$ cease to be consistent, and one must either change the model or introduce some mixing closure that stabilizes it. Similarly, when the model predicts wave-breaking, which will also mix the fluid, to continue using a shallow water model one must introduce additional mixing closures to complete the model $[8,9]$.

In this paper, however, we focus on the dynamics prior to breaking, and consequently neglect any mixing between the two fluid layers. 

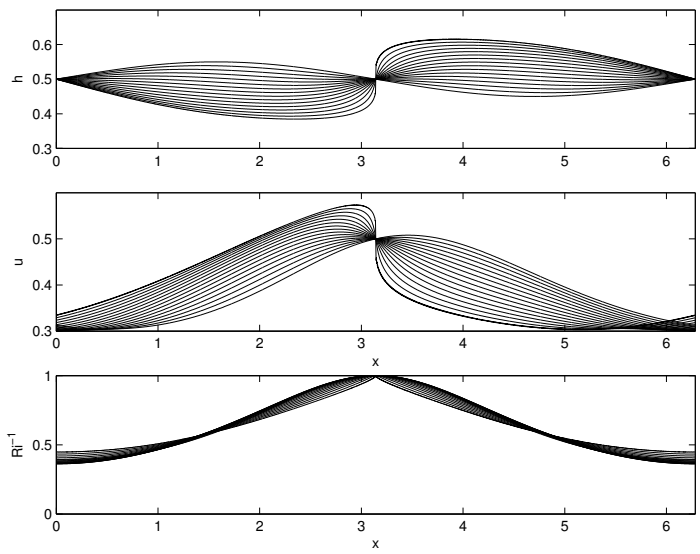

FIG. 3.2. Numerical solution of the system (2.18, 2.19), showing the evolution of $u, h$ and the computed $R i^{-1}$. The initial data was chosen to have $R i=1$ at one point. Note that the system never goes into the elliptic region, but that the wave breaks with "rollup" motion.
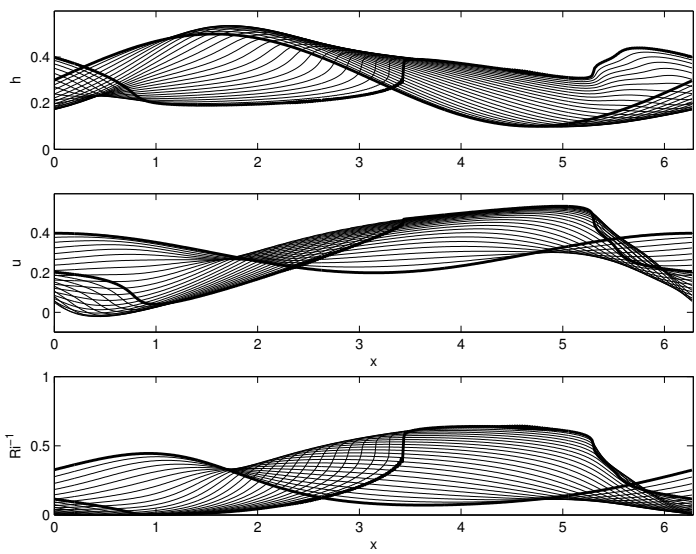

FIG. 3.3. Numerical solution of the system (2.18, 2.19), showing the evolution of $u, h$ and the computed $R i^{-1}$. Dark curves are the initial data and the solution at the last computed time.

\section{Expansion near criticality}

We shall now simplify the system $(2.18,2.19)$ further by expanding around a state with $R i=1$. This allows us to study the simplest possible problem near the hyperbolic-elliptic boundary (the sonic curve). Although this analysis will guide us in the right direction, our final results do not hinge on this expansion and apply to the full system. To this end, we propose the expansion

$$
\begin{aligned}
& h=h_{0}+\eta \\
& u=u_{0}+\mu,
\end{aligned}
$$

with $0<h_{0}<1, u_{0}=\left(1-h_{0}\right), \eta<<h_{0}$, and $\mu<<u_{0}$.

At the linear level, this expansion gives

$$
\eta_{t}+\left(1-h_{0}\right) \eta_{x}+h_{0} \mu_{x}=0
$$




$$
\mu_{t}+\left(1-3 h_{0}\right) \mu_{x}-h_{0} \eta_{x}=0,
$$

a "parabolic" system with just one real characteristic. The characteristic form follows from adding and subtracting the equations:

$$
\begin{aligned}
& (\eta+\mu)_{t}+\left(1-2 h_{0}\right)(\eta+\mu)_{x}=0 \\
& (\eta-\mu)_{t}+\left(1-2 h_{0}\right)(\eta-\mu)_{x}=-2 h_{0}(\eta+\mu)_{x}
\end{aligned}
$$

The solution to this system has

$$
(\eta+\mu)=F(x-c t)
$$

and

$$
(\eta-\mu)=-2 h_{0} t F^{\prime}(x-c t)+G(x-c t),
$$

where $c=1-2 h_{0}$ and $F$ and $G$ are arbitrary functions.

Let us now consider a weakly nonlinear situation close to the parabolic regime. Inspired by the structure of the parabolic problem, we shall use the variables $a=$ $(h+u-1)$ and $b=\left(h-u+1-2 h_{0}\right)$, in terms of which the system $(2.18,2.19)$ reads

$$
\left(\begin{array}{l}
a \\
b
\end{array}\right)_{t}+\frac{1}{2}\left(\begin{array}{cc}
1-\frac{1}{R i}+\frac{2-4 h}{\sqrt{R i}} & 1-\frac{1}{R i}-2 h+\frac{2 h}{\sqrt{R i}} \\
-1+\frac{1}{R i}+2 h+\frac{2 h}{\sqrt{R i}} & -1+\frac{1}{R i}+\frac{2-4 h}{\sqrt{R i}}
\end{array}\right)\left(\begin{array}{l}
a \\
b
\end{array}\right)_{x}=\left(\begin{array}{l}
0 \\
0
\end{array}\right)
$$

Expanding close to the parabolic regime, with $a$ and $b$ small, we have

$$
\begin{aligned}
h & =h_{0}+\frac{a+b}{2} \\
u & =1-h_{0}+\frac{a-b}{2} \\
\frac{1}{\sqrt{R i}} & =\frac{1-h_{0}+\frac{a-b}{2}}{1-h_{0}-\frac{a+b}{2}} \approx 1+\frac{a}{1-h_{0}} \\
\frac{1}{R i} & \approx 1+2 \frac{a}{1-h_{0}},
\end{aligned}
$$

so the system (4.9) reduces to

$$
\left(\begin{array}{l}
a \\
b
\end{array}\right)_{t}+\left(\begin{array}{cc}
1-2 h_{0}-\frac{1+h_{0}}{1-h_{0}} a-b & -a \\
2 h_{0}+\frac{2}{1-h_{0}} a+b & 1-2 h_{0}+a-b
\end{array}\right)\left(\begin{array}{l}
a \\
b
\end{array}\right)_{x}=\left(\begin{array}{l}
0 \\
0
\end{array}\right)
$$

We may now change the independent variables into $\left(x-\left(1-2 h_{0}\right) t, h_{0} t\right)$, and the dependent ones into $\left(a / h_{0}, b / h_{0}\right)$, to obtain

$$
\left(\begin{array}{l}
a \\
b
\end{array}\right)_{t}+\left(\begin{array}{cc}
-\frac{1+h_{0}}{1-h_{0}} a-b & -a \\
2+\frac{2}{1-h_{0}} a+b & a-b
\end{array}\right)\left(\begin{array}{l}
a \\
b
\end{array}\right)_{x}=\left(\begin{array}{l}
0 \\
0
\end{array}\right)
$$

The eigenvalues of the matrix in (4.11) are given by

$$
\lambda=-\left(\frac{h}{1-h_{0}} a+b\right) \pm \sqrt{-a\left(2+b+\frac{1-2 h_{0}}{\left(1-h_{0}\right)^{2}} a\right)} .
$$



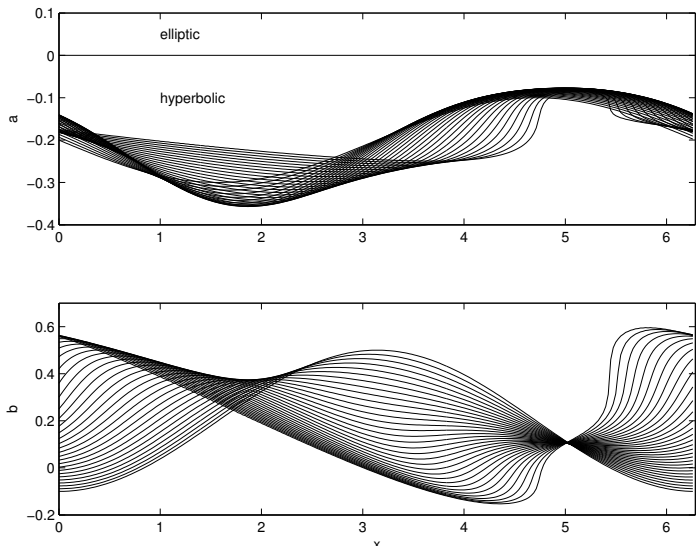

FIG. 4.1. Solution of the system (4.11), showing the evolution of $a, b$. Note that the system never goes into the elliptic region before breaking.
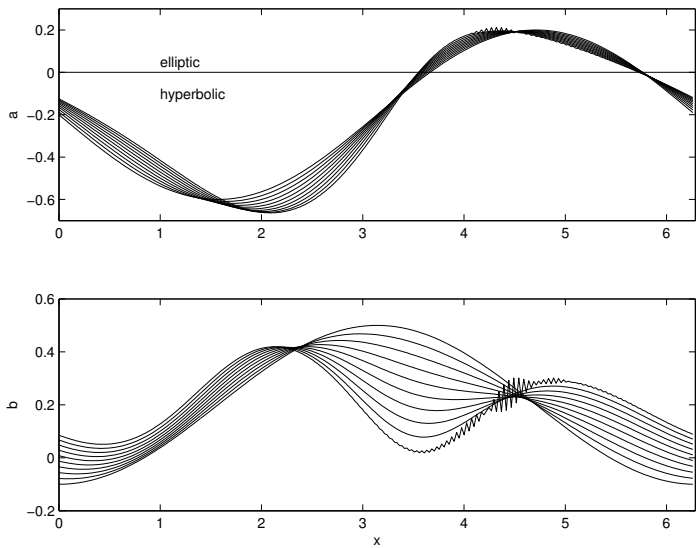

FIG. 4.2. Solution of the system (4.11), showing the evolution of a,b. The initial data was chosen to be partially in the elliptic domain. Note the numerical instability typical of attempting to numerically integrate an ill-posed problem.

Hence the condition for (4.11) to be hyperbolic is

$$
-a\left(2+b+\frac{1-2 h_{0}}{\left(1-h_{0}\right)^{2}} a\right)>0 .
$$

The only possibility consistent with the expansion giving rise to (4.11), which has $a$ and $b$ small, is $a<0$.

The numerical simulation displayed in figure 4.1 shows that this system does not cross the sonic boundary $a=0$. Solutions remain hyperbolic, with its waves deforming nonlinearly and eventually breaking. The solution shown in figure 4.2 starts in the elliptic domain, and exhibits typical instabilities of numerically computed elliptic initial value problems. If the grid is refined in this case, the grid-size oscillations would grow more rapidly since the problem is ill-posed. 

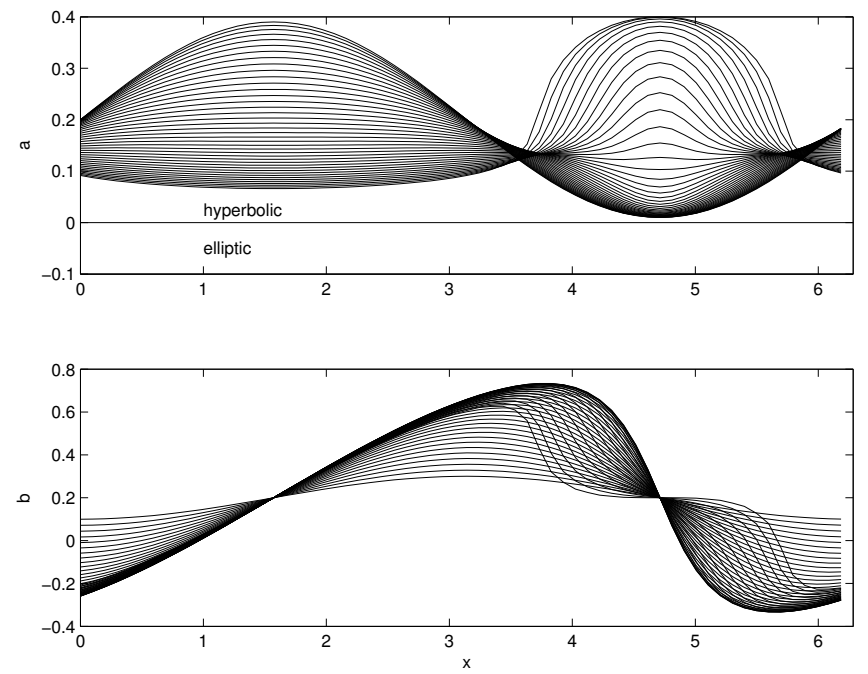

FIG. 5.1. Numerical solution of the model system (5.1). The solutions are shown up to $t=6$, which is close to breaking time. Note that the solution never crosses the sonic boundary $a=0$.

\section{Three simpler models}

To obtain a full understanding of why solutions cross or not into an elliptic region, we now introduce three simpler models that contain the essence of the problem. These simpler problems do not have particular physical relevance, but will illustrate the final results. First, a simple system that can be obtained from (4.11) by keeping only the dominant term in each equation with both $a$ and $b$ small:

$$
\begin{aligned}
a_{t}+a b_{x} & =0 \\
b_{t}+a_{x} & =0 .
\end{aligned}
$$

A contrasting example to (5.1) is the system

$$
\begin{aligned}
a_{t}+b b_{x} & =0 \\
b_{t}+a_{x} & =0 .
\end{aligned}
$$

The system (5.1) is hyperbolic for $a>0$ and elliptic for $a<0$ while the second system is hyperbolic for $b>0$ and elliptic for $b<0$.

Numerical calculations on these systems show that the first system remains hyperbolic, whilst the solution to the second system may cross the sonic boundary into the elliptic, unstable, domain. Figure (5.1) shows several snapshots of a typical solution to the system (5.1). The solution deforms and eventually breaks, but it never crosses the sonic boundary $a=0$. Figure (5.2) shows a typical solution to (5.2), crossing into the elliptic domain. Once in the unstable regime, numerical oscillations appear, with wavelength and growth rate determined by the numerical grid size.

A difference between the two systems that provides intuition for their different behavior is that, for the first model, sonic curves are characteristics, a condition that does not necessarily hold for the second.

The characteristic nature of the sonic curve for the system (5.1) can be derived as follows. At a sonic point, the characteristics have $d x / d t= \pm \sqrt{a}=0$. Along these 

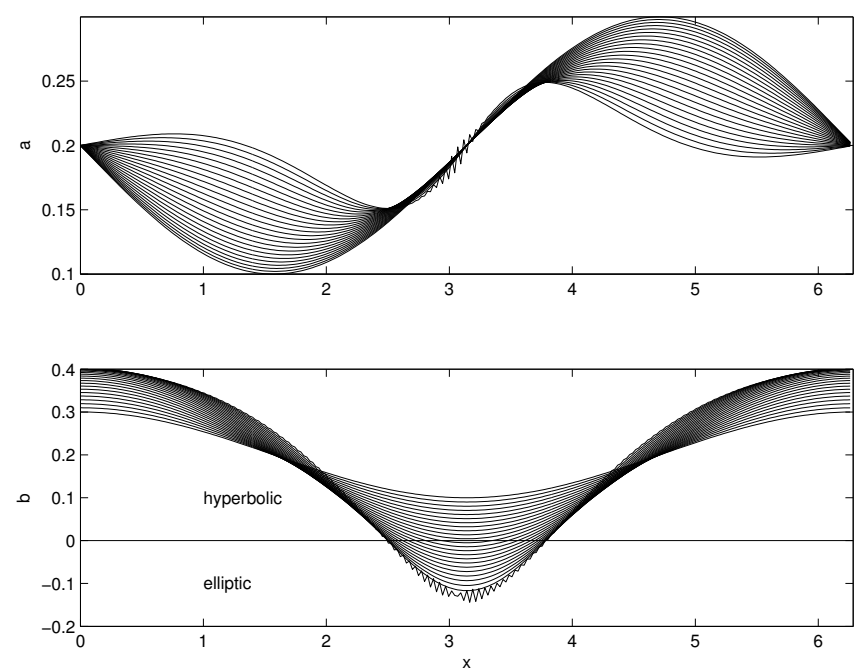

FIG. 5.2. Numerical solution of the model system (5.2). Note that the solution crosses the sonic boundary $b=0$, and exhibits the typical unstable numerical oscillations.

characteristics, $d a / d t=a_{t}+\frac{d x}{d t} a_{x}=a_{t}=-a b_{x}=0$. Hence the sonic condition $a=0$ is preserved along characteristics. The system (5.2), on the other hand, is sonic whenever $b=0$, where the characteristics have $d x / d t= \pm \sqrt{b}=0$. In this case, $d b / d t=b_{t}+\frac{d x}{d t} b_{x}=$ $b_{t}=-a_{x}$, which is not necessarily zero. Hence there exist non-characteristic sonic curves.

The hodograph transformation makes the tangency (or lack thereof) of the sonic curve and the characteristics particularly apparent (see appendix).

It is tempting to infer from these two examples that characteristic sonic curves are unreachable, while non-characteristic ones are not. Indeed the full two layer equations $(2.18,2.19)$ also share the property that sonic curves are characteristic. To see this, it is convenient to write the equations in terms of the variables $h$ and

$$
r=\frac{u}{1-h},
$$

whereby they become

$$
\begin{aligned}
h_{t}+r(1-2 h) h_{x}+h(1-h) r_{x} & =0 \\
r_{t}+\left(1-r^{2}\right) h_{x}+(1-2 h) r r_{x} & =0,
\end{aligned}
$$

with characteristics

$$
\frac{d x}{d t}=r(1-2 h) \pm \sqrt{h(1-h)\left(1-r^{2}\right)} .
$$

At the sonic boundary, the Richardson number $R i$ is one, so $r$ is either one or minus one, and

$$
\frac{d x}{d t}=r(1-2 h),
$$



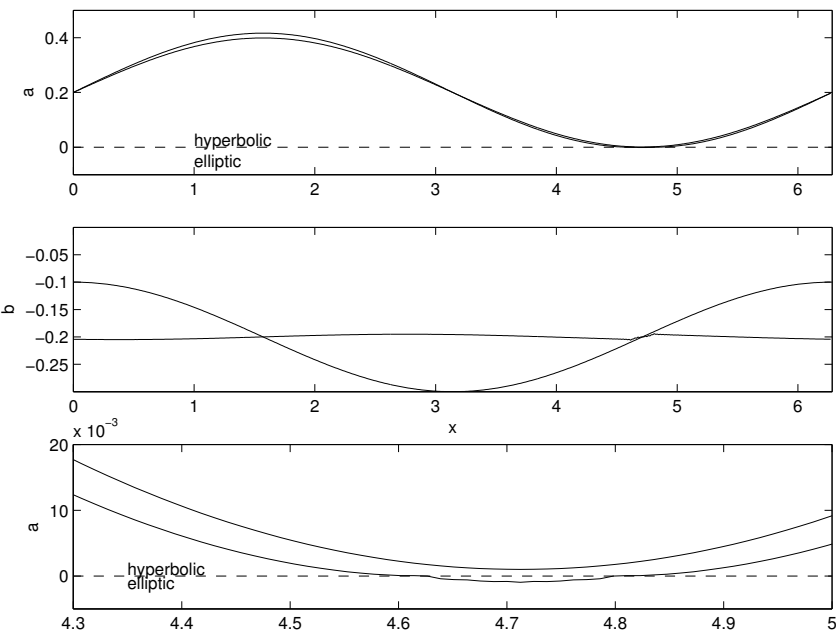

FIG. 5.3. Numerical solution of the model (5.8), with a close-up showing the crossing into the elliptic domain.

so along characteristics

$$
\frac{d r}{d t}=r_{t}+\frac{d x}{d t} r_{x}=0
$$

proving that the sonic condition is preserved.

That this condition is not sufficient in general, is clear from looking at a third simple model:

$$
\begin{aligned}
a_{t}+a^{1 / 3} b_{x} & =0 \\
b_{t}+a_{x} & =0 .
\end{aligned}
$$

Here the sonic line $a=0$ is also a characteristic, yet figure 5.3 displays a numerical solution crossing it from the hyperbolic into the elliptic domain.

A precise condition determining which systems permit crossings into the elliptic domain, follows from looking at the Riemann invariants of the three models. The characteristic form of the systems is

$$
R_{t}^{ \pm}+\lambda^{ \pm} R_{x}^{ \pm}=0 .
$$

For the system (5.1), the Riemann invariants are

$$
R^{ \pm}=b \pm 2 \sqrt{a},
$$

and the characteristic speeds are

$$
\lambda^{ \pm}= \pm \sqrt{a}= \pm \frac{1}{4}\left(R^{+}-R^{-}\right) .
$$

The systems (5.2) and (5.8) have

$$
\begin{aligned}
& R^{ \pm}=a \pm \frac{2}{3} b^{3 / 2} \\
& \lambda^{ \pm}=\mp \sqrt{b}=\mp \frac{3}{4}\left(R^{+}-R^{-}\right)^{\frac{1}{3}}
\end{aligned}
$$


and

$$
\begin{aligned}
& R^{ \pm}=b \pm \frac{6}{5} a^{5 / 6} \\
& \lambda^{ \pm}= \pm a^{\frac{1}{6}}= \pm \frac{5}{12}\left(R^{+}-R^{-}\right)^{\frac{1}{5}} .
\end{aligned}
$$

A difference between the system (5.1), which cannot reach the sonic line, and (5.2) and (5.8), than can, is that the characteristic slopes of the former are smooth functions of its Riemann invariants at the sonic points, which is not the case for the two others. As explained in the section below, this turns out to be the crucial distinction, providing a necessary and sufficient condition for hyperbolic-elliptic crossings.

As shown in [11], the characteristic structure (5.9) for the full two-layer system $(2.18,2.19)$ is surprisingly simple, with

$$
\begin{aligned}
R^{ \pm} & =2 \sqrt{h(1-h)\left(1-r^{2}\right)} \mp r(2 h-1) \\
\lambda^{ \pm} & = \pm \frac{3}{4} R^{ \pm} \mp \frac{1}{4} R^{\mp} .
\end{aligned}
$$

Hence the characteristics are smooth functions of the Riemann invariants, consistent with the numerical evidence indicating that the elliptic domain is unreachable.

\section{A general result for systems of 2 equations of mixed type}

Summarizing the evidence of the prior sections, we derive a result characterizing the possibility of smooth transitions into the elliptic, unstable domain:

Smooth solutions in the hyperbolic domain of a 2 X2 autonomous system of mixed type cannot reach the sonic boundary if the system's characteristic speeds are smooth functions of the Riemann invariants.

When this smoothness requirement is satisfied, a characteristic reaching a sonic point must have been sonic all along. Moreover, such a sonic characteristic must be a straight line in the $(x, t)$ plane, and the solution along it must be a constant. The smoothness of the characteristic fields forces sonic curves to be characteristic, but not the other way around: a system with characteristic sonic boundaries need not satisfy the smoothness requirement, and when smoothness is not present, sonic points may be reached. This explains why all examples where crossings are not observed have characteristic sonic boundaries, yet examples exist (i.e., model (5.8)), where characteristic sonic boundaries are not sufficient to inhibit crossings.

In particular, the two-layer shallow water model, our present physical application, satisfies the smoothness condition, and hence remains hyperbolic, at least up to breaking times.

To prove our statement, notice that the transition from hyperbolic to elliptic behavior occurs when the two characteristic slopes, $\lambda^{+}$and $\lambda^{-}$, real on the hyperbolic side, become identical on the sonic line, and then develop an imaginary component in the elliptic domain. Hence consider the evolution of their difference along a characteristic, say the $(+)$ one for concreteness. We have, with $d / d t=\partial / \partial t+\lambda^{+} \partial / \partial x$, that

$$
\frac{d\left(\lambda^{+}-\lambda^{-}\right)}{d t}=\frac{\partial\left(\lambda^{+}-\lambda^{-}\right)}{\partial R^{+}} \frac{d R^{+}}{d t}+\frac{\partial\left(\lambda^{+}-\lambda^{-}\right)}{\partial R^{-}} \frac{d R^{-}}{d t}
$$




$$
\begin{aligned}
& =\frac{\partial\left(\lambda^{+}-\lambda^{-}\right)}{\partial R^{-}}\left(\frac{\partial R^{-}}{\partial t}+\lambda^{-} \frac{\partial R^{-}}{\partial x}+\left(\lambda^{+}-\lambda^{-}\right) \frac{\partial R^{-}}{\partial x}\right) \\
& =\frac{\partial\left(\lambda^{+}-\lambda^{-}\right)}{\partial R^{-}}\left(\frac{\partial R^{-}}{\partial x}\right)\left(\lambda^{+}-\lambda^{-}\right)
\end{aligned}
$$

Since $\frac{\partial\left(\lambda^{+}-\lambda^{-}\right)}{\partial R^{-}}$is bounded by assumption (i.e., the $\lambda^{ \pm} \mathrm{S}$ depend smoothly on the $R^{ \pm} \mathrm{s}$ ), and if the solution remains smooth, we see that if the two characteristic slopes are initially different, they will remain different along the $(+)$ characteristic. It follows that, if the initial data is strictly hyperbolic, a smooth solution cannot reach the sonic boundary.

\section{Conclusions}

We have shown that long waves in two-layer flows can not give rise to profiles unstable to shear; these can only be imposed upon a flow by external forcing or boundary conditions. Instead, these waves will always deform and break, giving way to a mixing scenario quite different from the conventional one driven by shear-instability. How to actually model mixing due to internal breaking waves is a subject of active research, not addressed here.

In more generality, we have found a criterion for when a $2 \mathrm{X} 2$ autonomous system of mixed type can undergo a smooth hyperbolic-elliptic transition, leading to instability: such transitions are only possible in systems for which the dependence of the characteristic speeds on the Riemann invariants degenerate at sonic points, becoming non-smooth.

\section{Appendix A. Weakly nonlinear dynamics.}

Having established that the hyperbolic regime is an invariant set of the dynamics, it makes sense to study the weakly nonlinear propagation of waves [12]. Thus, in the hyperbolic regime, and using $\alpha=R^{-1}=\frac{u_{0}}{1-h_{0}}$, the linear waves have speed

$$
\lambda^{ \pm}=\alpha\left(1-2 h_{0}\right) \pm \sqrt{h_{0}\left(1-h_{0}\right)\left(1-\alpha^{2}\right)},
$$

where $|\alpha|<1$.

Defining $S=\sqrt{h_{0}\left(1-h_{0}\right)\left(1-\alpha^{2}\right)}$, and seeking a weakly nonlinear expansion of the form

$$
\begin{gathered}
h=h_{0}+\epsilon h_{0} \sigma\left(x-\lambda^{ \pm} t, \epsilon t\right)+\ldots \\
u=u_{0}+\epsilon\left( \pm S-\alpha h_{0}\right) \sigma\left(x-\lambda^{ \pm} t, \epsilon t\right)+\ldots
\end{gathered}
$$

one obtains the following Burger's equation for $\sigma(x, \tau)$ :

$$
(\mp 2 S) \sigma_{\tau}+R \sigma \sigma_{x}=0
$$

where

$$
R=\frac{-\left(S^{2} \mp 2 S \alpha h_{0}+\alpha^{2} h_{0}^{2}\right)\left(3-5 h_{0}\right)+4\left(\mp S-\alpha h_{0}\right) h_{0}^{2} \alpha+\left(1-h_{0}+2 \alpha^{2}\right) h_{0}^{2}}{1-h_{0}} .
$$

In the case of a shearless basic state, $u_{0}=0$, then $\alpha=0$, and we have

$$
\sigma_{\tau} \pm \frac{3}{2} \sqrt{\frac{h_{0}}{1-h_{0}}}\left(1-2 h_{0}\right) \sigma \sigma_{x}=0 .
$$



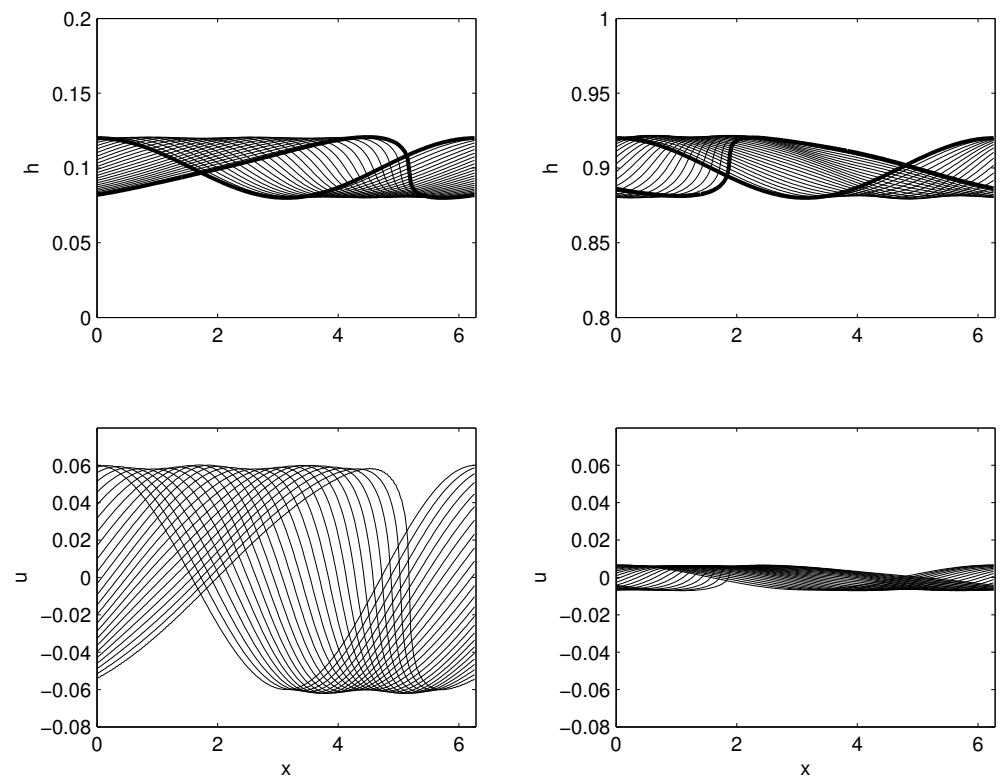

FIG. A.1. Numerical solution of the full equations (2.18, 2.19) in a weakly nonlinear regime where the solutions are governed approximately by Burgers equation (A). On the left is a case where the bottom fluid layer is thin and on the right, the top layer is thin. Note the direction of wave propagation and breaking.

This shows the expected symmetry $h_{0} \rightarrow 1-h_{0}$ and $\sigma \rightarrow-\sigma$ between waves of elevation and depression about $h_{0}=1 / 2$. Solutions of the full system $(2.18,2.19)$ in this regime are shown in figure A.1. For $h_{0}<1 / 2$, i.e. when the bottom layer is shallower that the top one, the waves break much as bores in single layer shallow water, with the lower layer's depth increasing as the bore passes through. Reciprocally, it is the upper layer which grows across bores when $h_{0}>1 / 2$.

\section{Appendix B. The hodograph plane.}

For our model systems $(5.1,5.2)$, the sonic curves in $(x, t)$ space become straight lines in the hodograph plane $(a, b)$, and the characteristic lines are easy to compute and draw. For the model (5.1), the hodograph transformation yields the linear system

$$
\begin{aligned}
X_{b}+a T_{a} & =0 \\
X_{a}+T_{b} & =0 .
\end{aligned}
$$

Eliminating $T$ yields the Keldysh equation

$$
X_{b b}-a X_{a a}=0 .
$$

Its characteristics satisfy

$$
\frac{d a}{d b}= \pm \sqrt{a}
$$

which can be integrated into

$$
a=\frac{1}{4}\left(b-b_{0}\right)^{2}
$$


Hence (see figure B.1), the characteristic lines are tangent to the sonic line $a=0$. In other words, the sonic line is also a characteristic.

For the model (5.2), on the other hand, the hodograph transform gives,

$$
\begin{aligned}
X_{b}+b T_{a} & =0 \\
X_{a}+T_{b} & =0 .
\end{aligned}
$$

which yields the Tricomi equation

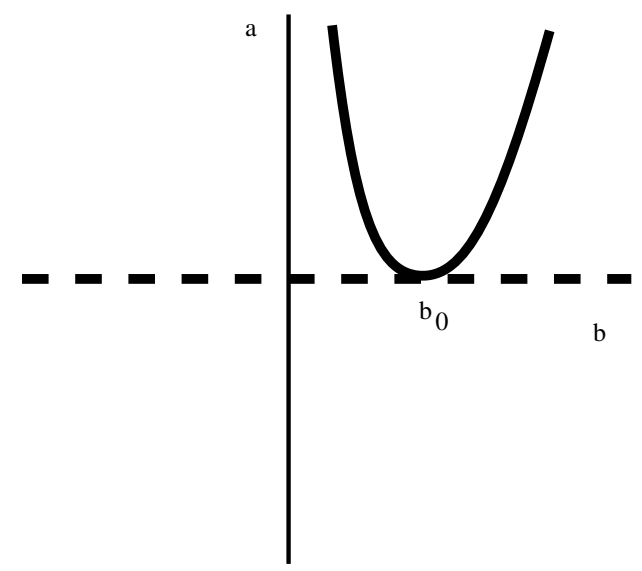

Fig. B.1. The sonic line is a characteristic

$$
T_{b b}-b T_{a a}=0
$$

The characteristic equations are

$$
\frac{d a}{d b}= \pm \sqrt{b}
$$

with solutions

$$
b=\frac{3}{2}\left(a-a_{0}\right)^{2 / 3} .
$$

These are not tangent to the sonic line $(b=0)$, which is therefore not a characteristic (see figure B.2). 


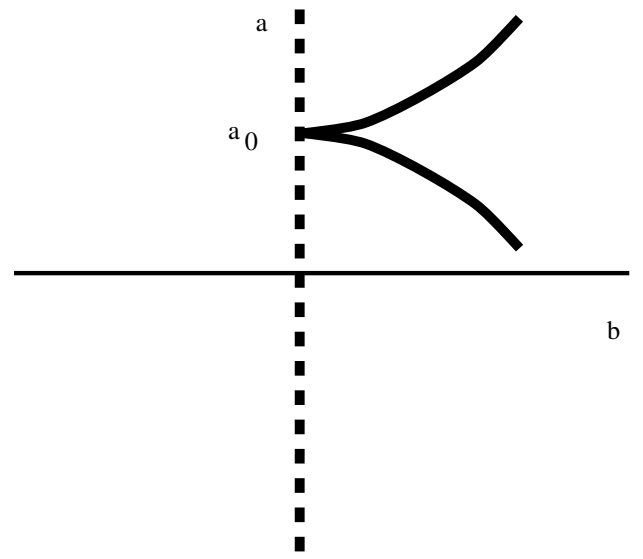

FIG. B.2. The sonic line is not a characteristic

Acknowledgments. The work of C. Turner and F. Menzaque was partially supported by a grant from the Fundación ANTORCHAS, and the work of Milewski and Tabak by a grant from the Division of Mathematical Sciences at the National Science Foundation.

\section{REFERENCES}

[1] A.D.D. Craik, Wave Interactions in Fluid Flows, Cambridge University Press, Cambridge, 1985.

[2] G. B. Whitham, Linear and Nonlinear Waves, Wyley Interscience, 1974.

[3] A. E. Gill, Atmosphere-Ocean Dynamics, Academic Press, 1982.

[4] R. W. Hallberg, Time integration of diapycnal diffusion and Richardson number dependent mixing in isopycnal coordinate ocean models, Mon. Wea. Rev., 128, 1402-1419, 2000.

[5] J. W. Miles, On the stability of heterogeneous shear flows, J. Fluid Mech., 10, 496-508, 1961.

[6] L. N. Howard, Note on a paper of John W. Miles, J. Fluid Mech., 10, 509-512, 1961.

[7] B. Cushman-Roisin, Introduction to Geophysical Fluid Dynamics, Prentice-Hall, 1994

[8] D. M. Holland, R. R. Rosales, D. Stefanica, and E. G. Tabak, Internal hydraulic jumps and mixing in two-layer flows, J. Fluid Mech., 470, 63-83, 2002.

[9] T. Jacobson, P. A. Milewski, and E. G. Tabak, A closure for mixing at internal breaking waves, in preparation.

[10] E. G. Tabak and F. A. Tal, Mixing in simple models for turbulent diffusion, CPAM, 57, 1-27, 2004.

[11] P. A. Milewski and E. G. Tabak, A simple formulation for fully nonlinear two-layer flows, in preparation.

[12] D. J. Benney, Long non-linear waves in fluid flows, J. Math. and Phys., 45, 52-63, 1966. 